\title{
Molecular imaging and depth profiling of biomaterials interfaces by femtosecond laser desorption postionization mass spectrometry
}

\section{Authors: Yang Cui, Chhavi Bhardwaj, Slobodan Milasinovic, Ross P. Carlson, Robert J. Gordon, and Luke Hanley.}

NOTICE: This document is the unedited author's version of a Submitted Work that was subsequently accepted for publication in ACS Applied Materials \& Interfaces, copyright (C) American Chemical Society after peer review. To access the final edited and published work, see http://dx.doi.org/10.1021/am4020633.

Cui Y, Bhardwaj C, Milasinovic S, Carlson RP, Gordon RJ, Hanley L, "Molecular imaging and depth profiling of biomaterials interfaces by femtosecond laser desorption postionization mass spectrometry," ACS Applied Materials \& Interfaces October 2013, 5 (19):9269-9275. 


\title{
Molecular Imaging and Depth Profiling of Biomaterials Interfaces by Femtosecond Laser Desorption Postionization Mass Spectrometry
}

\author{
Yang Cui ${ }^{\dagger}$ Chhavi Bhardwaj, ${ }_{, j}^{\dagger}$ Slobodan Milasinovic, ${ }^{\dagger}$ Ross P. Carlson, ${ }^{\dagger}$ Robert J. \\ Gordon, ${ }^{\top}$ and Luke Hanley*, ${ }^{\dagger}$ \\ †Department of Chemistry, University of Illinois at Chicago, Chicago, Illinois 60607, United States \\ \$Center for Biofilm Engineering, Montana State University, Bozeman, Montana 59717, United States
}

\section{ABSTRACT:}

Mass spectrometry (MS) imaging is increasingly being applied to probe the interfaces of biomaterials with invasive microbial biofilms, human tissue, or other biological materials. Laser desorption vacuum ultraviolet postionization with $\sim 75 \mathrm{fs}, 800 \mathrm{~nm}$ laser pulses (fs-LDPI-MS) was used to collect MS images of a yeast-Escherichia coli co-culture biofilm. The method was also used to depth profile a three-dimensionally structured, multispecies biofilm. Finally, fs-LDPI-MS analyses of yeast biofilms grown under different conditions were compared with LDPIMS using ultraviolet, nanosecond pulse length laser desorption as well as with fs laser desorption ionization without postionization. Preliminary implications for the use of fs-LDPI-MS for the analysis of biomaterials interfaces are discussed and contrasted with established methods in MS imaging.

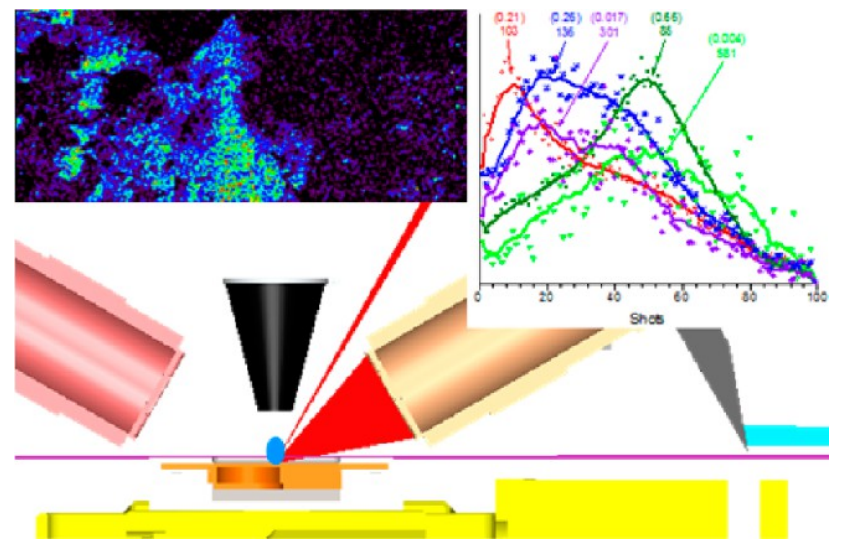

KEYWORDS: laser desorption, femtosecond, mass spectrometry, imaging, biofilm, single photon ionization

\section{INTRODUCTION}

Mass spectrometric (MS) imaging is increasingly being used for the spatially resolved molecular analysis of the interfaces of biomaterials with invasive microbial biofilms, human tissue, and other biological materials. ${ }^{1}$ The methods used for MS imaging include secondary ion mass spectrometry (SIMS), ${ }^{2}$ as well as those based on electrospray ionization and matrix-assisted laser desorption ionization (MALDI) using nanosecond (ns) pulse length lasers. ${ }^{1,3-6}$ Despite its popularity and numerous advantages, MALDI has several shortcomings when used for MS imaging of molecular species from biomaterials interfaces. Sample preparation often requires multiple steps including tissue washing and multiple matrix applications to enhance ion yields. ${ }^{3,6,7}$ MALDI displays overall low ion yields, with typically $1 \times 10^{3}$ to $1 \times 10^{7}$ neutrals desorbed along with every ion. ${ }^{8-10}$ Salts, sample-specific ion suppression, and interferences between multiple analytes lead to strong dependence of ionization efficiency on both analyte and local chemical environment. ${ }^{3}$ These effects collectively limit the classes of species readily detected by MALDI and its capability for quantification.

This work describes the use of laser desorption with ultrashort pulse lasers for MS imaging, a method that differs in several fundamental ways from MALDI with ns pulsed lasers. Specifically, sub-100 fs, $\sim 800 \mathrm{~nm}$ laser pulses induce a nonresonant desorption event that does not require any addition of matrix compounds (unlike MALDI). Furthermore, UV ns lasers leave chemical damage after sampling, preventing same-spot reanalysis for depth profiling in MALDI-MS. However, fs laser ablation can remove sample from a solid while leaving minimal damage to the remaining material, a remarkable characteristic that has motivated fs laser applications in surgery. ${ }^{11}$

-14 MS analysis before and after ablation with fs laser pulses has verified the removal of material for depth profiling while allowing re-analysis at the same spot. ${ }^{7,15}$ Finally, fs laser desorption ionization (fs-LDI-MS) has been demon-strated for analysis of solid samples by the direct formation of gaseous ions. ${ }^{16-20}$ Some of the authors recently reported the development of a fs-LDI-MS instrument with imaging capabilities. 21

Ultrashort pulse laser ablation has also been used to desorb neutrals for electrospray postionization. ${ }^{22-25}$ Vacuum ultra-violet (VUV) single photon ionization is another postionization method that has been coupled to UV ns laser desorption. ${ }^{26-30}$ VUV single photon ionization of isolated molecules in vacuum occurs only when their ionization energies are below that of incident photons, rendering $10.5 \mathrm{eV}$ VUV radiation sufficient for a wide range of analytes. $31-33$ Sensitivity in VUV single photon ionization in vacuum depends on the ionization yield to produce the radical cation $\mathrm{M}^{+}$. The ion yield $\mathrm{Y}=\sigma_{\text {spi }} \mathrm{IN}_{\text {gas }}$, where $\sigma_{\mathrm{spi}}$ is the photoionization cross section at a given photon 




Figure 1. Schematic diagram of femtosecond laser desorption postionization mass spectrometer (fs-LDPI-MS) showing the $10.5 \mathrm{eV}$ (118 nm) VUV generation cell, optical layout, UV laser beam blocker, VUV diagnostic detector, and reflectron time-of-flight MS assembly.

energy $h \nu, I$ is the intensity of VUV radiation, and $N_{\text {gas }}$ is the density of gaseous neutrals. ${ }^{29}$ This linear relationship of ion yield to gaseous neutral density has allowed ns laser desorption combined with $7.87 \mathrm{eV}$ single photon ionization (ns-LDPI-MS) to be used for quantification of an antibiotic infused into a drug delivery multilayer. ${ }^{34}$

Ns-LDPI-MS with $7.87 \mathrm{eV}$ radiation has been used to detect derivatized peptides ${ }^{28}$ and select antibiotics in microbial biofilms. ${ }^{31,34,35}$ Although $7.87 \mathrm{eV}$ energy photons ionize only low ionization energy species such as fused ring systems and tertiary amines, the high intensity of the molecular fluorine laser saturates photoionization to achieve maximum detection of neutrals with low background signal. Higher photon energy sources such as those at $10.5 \mathrm{eV}$ can detect a wide range of laser-desorbed species such as endogenous species in biofilms, ${ }^{31,36}$ although the relatively low intensity of such VUV sources does not allow saturation of photoionization.

The addition of $10.5 \mathrm{eV}$ postionization and other improvements to the fs-LDI-MS instrument ${ }^{21}$ permit spatially resolved molecular analysis of intact biological samples, which is demonstrated here on several microbial biofilms grown under different conditions. These samples serve as models of biomaterials interfaces and are used for a preliminary comparison fs-LDI-MS and ns-LDPI-MS with fs-LDPI-MS. One of the biofilm systems is also used to demonstrate depth profiling with the latter.

\section{EXPERIMENTAL SECTION}

Figure 1 shows a schematic diagram of the fs-LDPI-MS that is based upon a previously reported fs-LDI-MS instrument. ${ }^{21}$ A $800 \mathrm{~nm}, \sim 45 \mathrm{fs}$ pulse length ultrafast laser beam from a Ti:sapphire laser was focused onto a sample by an achromatic doublet lens, with a delivered pulse width of $\sim 75$ fs. Samples were mounted on a 3D motion stage with submicrometer positioning accuracy. Ion optics consisted of Einzel lenses, deflectors, pulsing electrodes, and a linear time-of-flight (TOF) drift tube. A digital single lens reflex (DSLR) camera and beamsplitter were used to optically image the sample. Several major modifications have been made to the instrument, including addition of a second camera, a new objective for the fs laser, improved focusing optics, a $10.5 \mathrm{eV}$ photon energy source, and a reflectron TOF (reTOF). The previously reported ion funnel was removed temporarily because it does not improve the data quality in the low pressure experiment described here. Other changes to the instrument are noted below.

Optics. Two optical beam paths were used. One, described previously, ${ }^{21}$ consisted of a long working distance objective (10X NA 0.28 , Mitutoyo, Japan) with a $60^{\circ}$ incidence angle with respect to the sample normal. The second beam path used an achromatic doublet lens (200 mm focal length, Thorlabs) with a $30^{\circ}$ incidence angle. Switching between the two beam paths was achieved by demountable magnetic kinematic mount (Thorlabs). A $2 \times$ Galileo-type beam expander was inserted into the beam upstream to reduce the laser focus size on the sample. The $30^{\circ}$ fs laser focusing optics provided a focused beam diameter of $\sim 25 \mu \mathrm{m}$ and a Rayleigh range of $\sim 100 \mu \mathrm{m}$, which is better suited for rough surfaces such as dried biofilms. The $60^{\circ}$ objective focused the fs laser beam to a $7 \mu \mathrm{m}$ diameter and was used for high spatial resolution. To further improve the beam quality, the previously described double-pulse setup and dichroic beamsplitter were bypassed. ${ }^{21}$ Optical imaging was done using a separate objective, as illustrated in Figure 1.

10.5 eV VUV Generation. A $10.5 \mathrm{eV}$ photon energy source was built and installed on the instrument for VUV postionization, employing a design that was improved from the third harmonic generation gas cell described previously. ${ }^{36}$ The $355 \mathrm{~nm}$ output of a ns Nd:YAG laser (Tempest-10, New Wave Research, Portland, OR) was used to pump a Xe/Ar frequency tripling cell. A beam sampler was also inserted in the beam path, extracting $6 \%$ the beam for monitoring the pulse energy. A UV coated $250 \mathrm{~mm}$ focal length fused silica lens (PLCX-38.1-128.8-UV-355, CVI, Albuquerque, NM) was mounted on a detachable viewport flange (112667, Accuglass, Valencia, CA) for isolating the gas mixture from the atmosphere and focusing the beam to generate VUV radiation. An off-axis LiF lens (Beijing Scitilon, China) was used as a VUV collimation lens. It was also used as a dispersion element to separate and block the $355 \mathrm{~nm}$ primary beam by an aluminum beam blocker.

Use of an antireflection coated quartz lens as a window transmitted more $355 \mathrm{~nm}$ radiation into the system, as compared to the previous design which lost $\sim 20 \%$ of the pump laser power by reflection from quartz windows. Fixing the lens to the cell also decreased the possibility of laser damage.

A simple photoelectron current detector was built based on a published design ${ }^{37}$ and mounted opposite the gas cell for the purpose of monitoring VUV generation and assisting beam alignment. The detector consisted of a $\mathrm{Ni}$ plate having an $\sim 5 \mathrm{eV}$ work function that allowed generated photoelectrons to be extracted from the cathode by a $+250 \mathrm{~V}$ biased Ni mesh anode and monitored by an oscilloscope (TDS5104S, Tektronix, Beaverton, OR). The VUV detector had an $\sim 1 \times 10^{4}$ discrimination of VUV vs. UV photoelectron current. 
Although no attempt was made to calibrate the detector to obtain absolute VUV flux, it was confirmed that the relationship between VUV flux and photoelectron current depended only on the electrical field strength and the VUV beam size.

Mass Analyzer. A two-stage reflectron TOF was scavenged from a donated instrument (Pegasus III, LECO) and coupled to the fs-LDPIMS after the detector (31374, Photonis, Lancaster, PA) was refurbished. High voltage power supplies (PMT series, Bertan/ Spellman, Hauppauge, NY) removed from this instrument were reused for the reflectron with customized controlling circuits. A conical nipple vacuum adapter was used to couple the TOF drift tube to the instrument main chamber. The reTOF had a screen inside the vacuum tube chamber that could be biased negatively but was grounded for these experiments. Because the ion source was located outside of the reflectron's focal point, a second-order energy correction could not be achieved. Nevertheless, mass resolution up to $\sim 1100$ was demonstrated in both LDI and LDPI modes, in agreement with ion optical simulations (SIMION 8.1, Scientific Instrument Services, Ringoes, $\mathrm{NJ}$ ).

LDI ions were mostly removed from LDPI mass spectra by pulsing a deflector to $+400 \mathrm{~V}$ when firing the desorption laser. Several $\mu$ s after firing the VUV-generating Nd:YAG laser, the deflector voltage was pulled down to ground to shunt all photoions into the re-TOF. There was also a balance between the upper $\mathrm{m} / \mathrm{z}$ limit of direct ions rejected and lower $\mathrm{m} / \mathrm{z}$ limit of photoions passed to the detector, which was determined by the delay between Nd:YAG firing and the falling edge of the deflector bias. The typical delay used was chosen such that direct ion rejection was up to $\mathrm{m} / \mathrm{z} \sim 300$ and photoions transmitted down to $\mathrm{m} / \mathrm{z} \sim 50$. The deflector could also be placed closer to the ion source so that the direct ion rejection would extend above $\mathrm{m} / \mathrm{z} \sim 1000$ while allowing photoions above $\mathrm{m} / \mathrm{z} \sim 50$ to pass into the reTOF.

A gas mixture consisting of acetone, hexane, toluene, octane, and $\alpha$ pinene was leaked into the vacuum chamber and detected by photoionization as a complementary check of VUV generation and additionally for mass calibration. In most cases, an accurate calibration with the aforementioned molecules could be extrapolated to $\mathrm{m} / \mathrm{z} 500$ with less than $\mathrm{m} / \mathrm{z} 1$ mass shift. Higher molecular weight molecules such as $\mathrm{C}_{60}$ and sexithiophene were used to calibrate the higher mass range.

Ns-LDPI-MS. Comparison with ns-LDPI-MS was performed by analysis of samples using a separate custom-made instrument equipped with a $\sim 5$ ns diode-pumped solid state (DPSS) Nd:YLF (Explorer, Spectra-Physics) desorption laser, a Nd:YAG (Tempest-10, New Wave Research, ESI, Portland, OR) ionization laser to pump a $10.5 \mathrm{eV}$ VUV frequency tripling cell filled with pure $\mathrm{Xe}$, and a custom reTOF used for mass analysis. ${ }^{36}$ Sample positioning and scanning were done by a two-dimensional vacuum-compatible motion stage, similar to that employed in the fs apparatus, capable of submicrometer precision.

Data Acquisition and Analysis Software. The instrument control and data processing software have been further modified from the previous description. ${ }^{21} \mathrm{~A}$ simple filter was used to increase the signal-to-noise ratio of the MS data, in which a threshold was set by the software to remove baseline electronic noise by setting a data point to zero if it dropped below a 5-10 mV threshold. Further processing of mass spectra was performed by importing raw data files into commercial data analysis software (OriginPro, OriginLab, Northampton, MA). For imaging and depth profiling, in-house software was used to process and convert binary files to compatible formats for input into other software (i.e., BioMAP, www.maldi-msi.org) ${ }^{21}$ The analysis tools were also revised for re-calibrating mass spectra, peak searching, and centroiding.

Sample Preparation. Baker's yeast Saccharomyces cerevisiae (Red Star Yeast Company) monoculture biofilms were grown either on polycarbonate membranes (GE PCTE filter membranes, 09-732-18, Fisher Scientific) in sessile media ${ }^{36}$ or on indium tin oxide (ITO) coated glass or medical titanium (Ti-6Al-4V, 9081K111, McMaster) in a drip flow biofilm reactor. ${ }^{31}$ Yeast-Escherichia coli (ATCC 25922, American Type Culture Collection, Manassus, VA) coculture biofilms were grown on polycarbonate membranes. ${ }^{36} \mathrm{~A}$ chronic wound model biofilm system was comprised of medical isolates of the bacteria
Staphylococcus aureus (MRSA), Pseudomonas aeruginosa, and Clostridium perfringens. This polymicrobial mixture was grown on polycarbonate membranes placed on brain heart infusion (BHI) agar media. ${ }^{38}$ Membrane biofilms were attached to stainless steel MALDI sample plates by copper tape or blotted for analysis by LDPI-MS, as noted below. Drip flow reactor biofilms were analyzed directly.

\section{RESULTS AND DISCUSSION}

Comparison of fs-LDI-MS and fs-LDPI-MS. Figure 2 shows a comparison between fs-LDI and fs-LDPI mass spectra

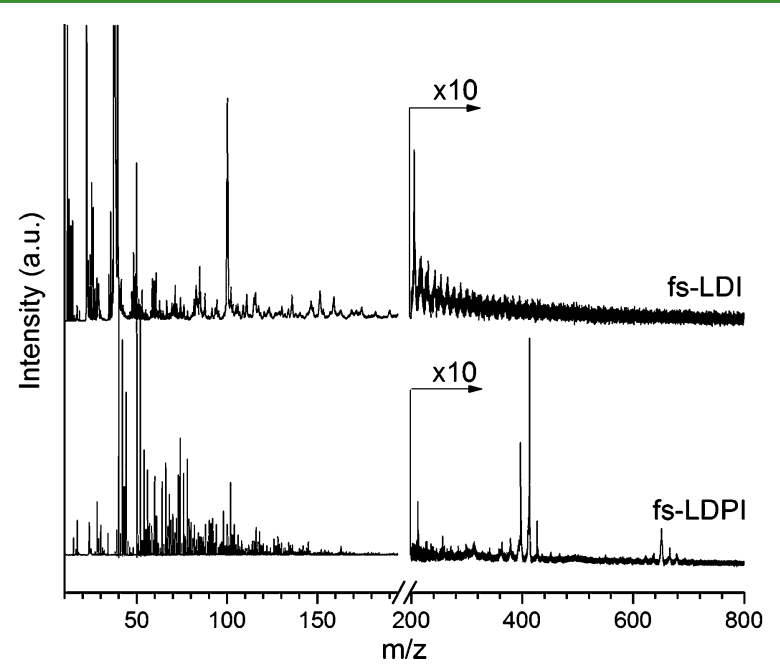

Figure 2. Comparison between fs laser desorption ionization (fs-LDI) and fs-LDPI-MS of yeast biofilms grown on polycarbonate (PC) membranes, with the fs-LDI-MS signal scaled by $20 \times$. The VUV source was turned off to collect fs-LDI-MS.

of yeast biofilms grown on polycarbonate (PC) membranes. The fluence of the desorption laser was $\sim 0.1 \mathrm{~J} / \mathrm{cm}^{2}$ for fs-LDI mass spectra, $\sim 2-3 \times$ above the ion formation threshold, chosen to achieve a reasonable signal-to-noise ratio without the dramatic degradation of mass resolution observed at higher fluences. The desorption fluence for fs-LDPI was $\sim 0.3 \mathrm{~J} / \mathrm{cm}^{2}$, $\sim 2 \times$ above the desorption threshold. Fs-LDPI employed a pulsed deflector to reject ions formed by direct LD, whereas the same deflector was simply grounded in fs-LDI mode.

Figure 2 clearly shows that fs-LDPI-MS generated much more molecular information than fs-LDI-MS above $\mathrm{m} / \mathrm{z} 300$ (see below). The peak patterns are notably different for the two detection methods. The fs-LDPI signal was $\sim 20 \times$ higher than for fs-LDI. The most intense peaks in fs-LDI-MS are due to $\mathrm{Na}^{+}$and $\mathrm{K}^{+}$ions.

Comparison of ns vs. fs Laser Desorption. The bottom two spectra in Figure 3 of the same type of yeast biofilms grown on PC membranes show the difference between ns and fs laser desorption neutrals that are photoionized by $10.5 \mathrm{eV}$ radiation. Both spectra show distinct peak patterns, which may be due to laser pulse length-dependent differences in desorption mechanism ${ }^{7}$ and/or internal energy transfer. ${ }^{36}$ Studies are underway to probe the extent of energy transfer during fs-LDI and fsLDPI-MS. The mass detection range was similar in both ns and fs-LDPI, ranging up to $\mathrm{m} / \mathrm{z} 800$.

Comparison of Different Substrates. Figure 3 displays fs-LDPI-MS of yeast membrane biofilms as well as drip flow biofilms grown on Ti and ITO glass. Some common peaks were observed, but overall spectra differed with substrate, especially at the lower mass range. Titanium gave the lowest signal of the 


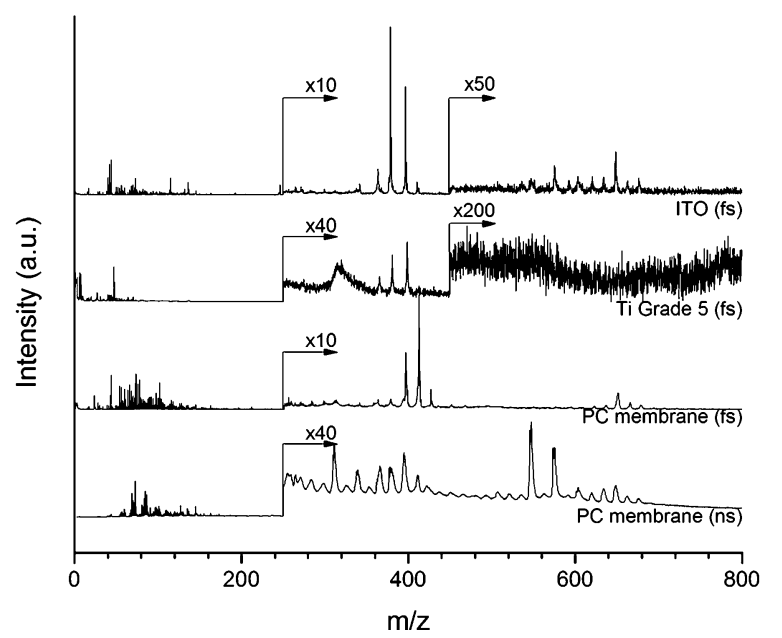

Figure 3. Comparison of ns- and fs-LDPI-MS of yeast biofilms grown on PC membranes, labeled "PC membrane (ns)" and "PC membrane (fs)", respectively, shown in the bottom two spectra. Fs-LDPI-MS of drip flow yeast biofilms grown on Ti and ITO glass surfaces are shown in the top two spectra.

three yeast biofilms by fs-LDPI, even though electrically conductive substrates improve ion extraction in the instrument. Peaks around $\mathrm{m} / \mathrm{z} 400$ displayed different ratios, which were possibly caused by metabolite differences. The biofilm grown on the ITO glass substrate displayed two unique peaks slightly above $\mathrm{m} / \mathrm{z} 110$ that could derive from indium and an organic species complexed with this constituent of ITO glass.

MS Imaging. The optical micrograph at the bottom of Figure 4 of yeast-E. coli coculture biofilms grown on membranes displayed three distinct regions attributed to "pure" yeast culture, "pure" E. coli, and a mixed region. MS images of the cocultured biofilms were recorded from a sample area of $7 \times 3 \mathrm{~mm}^{2}$ with desorption laser fluence of $0.5 \mathrm{~J} / \mathrm{cm}^{2}$, with two spectra recorded and averaged for each pixel. The imaging process took one hour to complete and was rate limited by the $10 \mathrm{~Hz}$ laser used to generate VUV radiation. The top five panels of Figure 4 display the MS images for total ion count, $\mathrm{m} / \mathrm{z} 93, \mathrm{~m} / \mathrm{z} 283, \mathrm{~m} / \mathrm{z} 258$, and $\mathrm{m} / \mathrm{z} 414$. The $\mathrm{m} / \mathrm{z} 93$ appeared mainly in the mixed region while $\mathrm{m} / \mathrm{z} 283$ only existed in yeast, and $\mathrm{m} / \mathrm{z} 258$ only in E. coli. The $\mathrm{m} / \mathrm{z} 258$ peak was previously attributed to a metabolite and was used to image different strains of E. coli by ns-LDPI-MS. ${ }^{36}$ The $\mathrm{m} / \mathrm{z} 414$ appeared mainly in the mixed region and also in the yeast region with lower abundance.

Depth Profiling. Previous studies showed the feasibility of using <100 fs, $800 \mathrm{~ns}$ laser desorption for depth profiling by continuously sampling a fixed spot area. ${ }^{7,15,21}$ The feasibility of depth profiling by fs-LDPI-MS is examined here using a chronic wound model membrane biofilm. This 3D biological model was developed previously from three bacterial species isolated from a medical infection that segregate vertically in a biofilm into aerobic (top) and anaerobic (substrate) regions. ${ }^{38} \mathrm{~A}$ depthprofiling experiment was performed in which the lasers ran for a total of $20 \mathrm{~s}$ (i.e., 200 laser shots), drilling a hole down into the intact biofilm sample, where each fs desorption laser shot was followed after $\sim 10 \mu \mathrm{s}$ delay by a VUV laser pulse. Each spectrum from the digitizer was stored separately, with no averaging, so that $\sim 200$ spectra were recorded for each hole. To improve the statistics, we repeated the measurement on 50 separate spots on the biofilm. All the data were sorted and

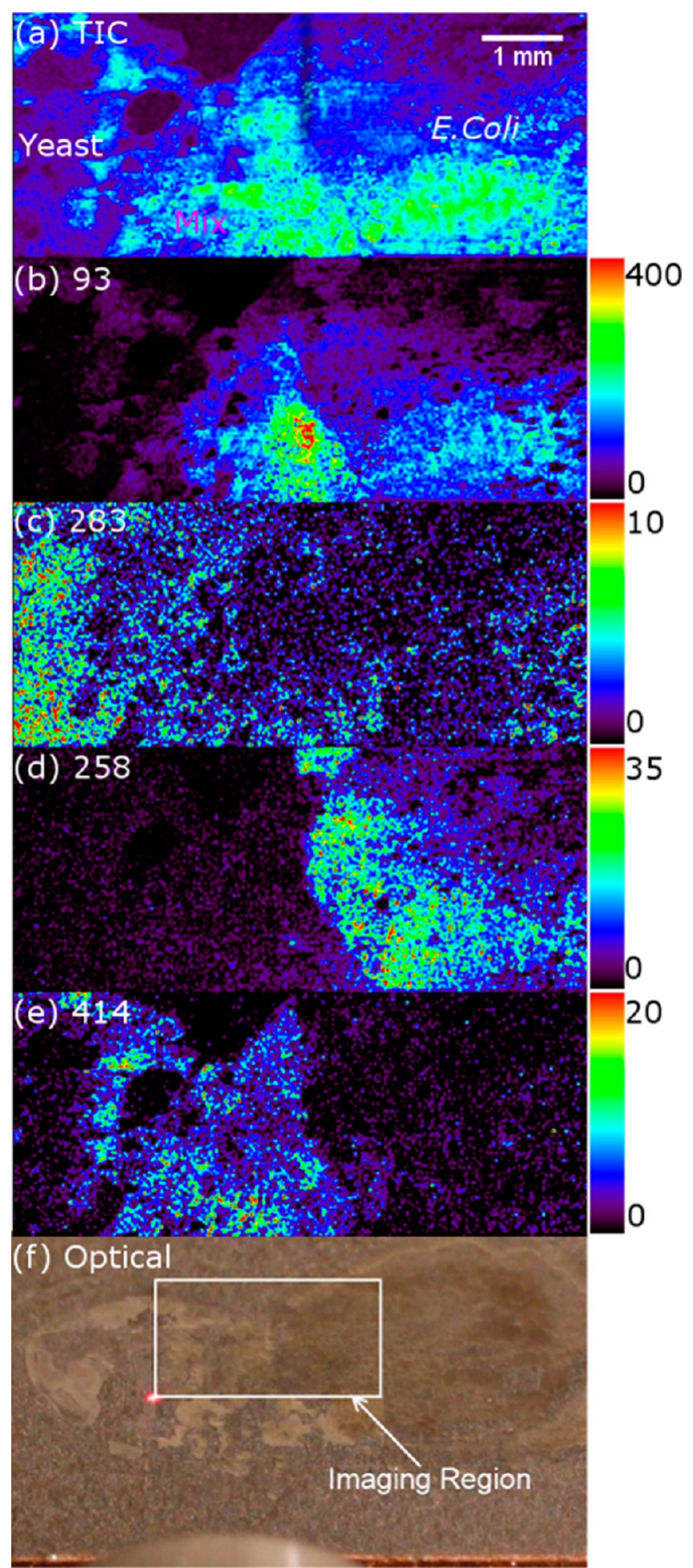

Figure 4. Ion images from fs-LDPI-MS of blotted co-cultured yeast-E. coli biofilm: (a) total ion current, (b) $\mathrm{m} / \mathrm{z}$ 93, (c) $\mathrm{m} / \mathrm{z} 283$, (d) $\mathrm{m} / \mathrm{z}$ 258, (e) $\mathrm{m} / \mathrm{z} 414$, and (f) optical image. Color bar on right indicates signal levels.

averaged according the number of shots at each point. ${ }^{21}$ Finally, the normalized peak areas corresponding to each peak were plotted in Figure 5 vs. the number of laser shots, corresponding approximately to the depth.

Three major profiles were observed from the depth profile of the chronic wound model biofilm, together with some peaks 


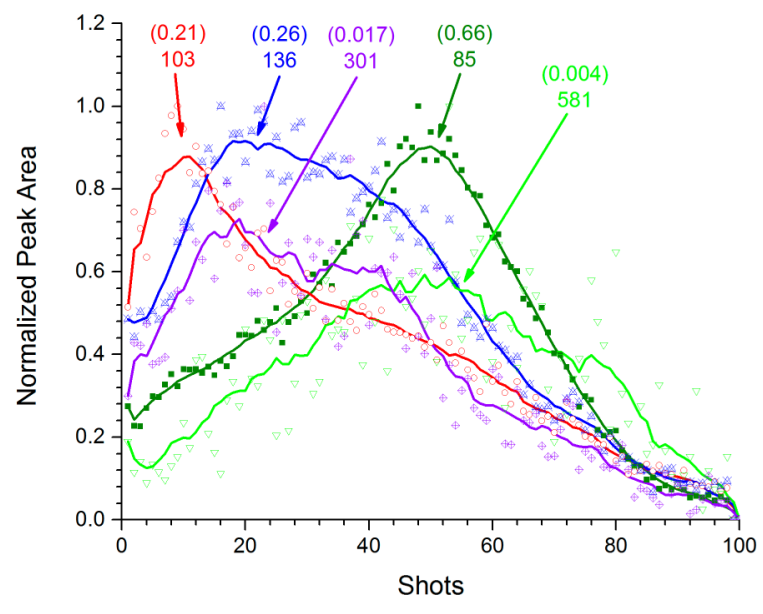

Figure 5. Depth profile of a polymicrobial chronic wound model membrane biofilm. Figure shows normalized peak areas of five

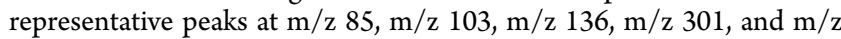
581 vs. depth as represented by the number of laser shots. Raw data points were smoothed by 10 point window averaging then plotted as continuous curves. Absolute peak areas are in parentheses.

that showed no intensity difference vs. depth. Figure 5 shows five peaks out of $\sim 15$ total observed, which displayed different intensities vs. depth. Because the absolute intensity of these peaks varied significantly, the data were normalized, and a 10 point window average smoothing was applied.

The chronic wound model consists of three medically isolated microbes which spatially segregate based on metabolic potential. $P$. aeruginosa resides at the aerobic top of the biofilm, where $\mathrm{m} / \mathrm{z} 103,136$, and 301 are the most intense in the Figure 5 depth profile. The middle region of the biofilm consisted largely of cell debris, where $\mathrm{m} / \mathrm{z} 301$ remained intense. C. perfringens and $S$. aureus resided near the anaerobic bottom of the biofilm where $\mathrm{m} / \mathrm{z} 85$ and 551 were most intense (near laser shot 50). All peaks decreased beyond laser shot 80, indicating that the biofilm was mostly removed. Figure 5 accordingly displays data from only the first 100 laser shots. Although these results support the feasibility of depth profiling by fs-LDPI, correlating analyses are required to confirm that the different profiles measured do in fact match the microbial spatial distributions.

Spatial Resolution and Other Instrumental Issues. The instrument was also equipped with an objective capable of delivering a $7 \times 8 \mu \mathrm{m}^{2}$ focal spot. A spatial resolution of $\sim 10$ $\mu \mathrm{m}$ was previously demonstrated using an inorganic test target with a laser focus size of $32 \times 16 \mu \mathrm{m}^{2}$ caused by the nonlinear ablation event. ${ }^{21}$ Analyses of biofilms by fs-LDPI-MS, however, have not yet produced such high spatial resolution. One reason is the relatively short Rayleigh range of $\sim 100 \mu \mathrm{m}$ for the microfocused fs laser beam. Such short Rayleigh ranges were problematic for the biofilms studied here given that they displayed surface roughnesses $>100 \mu \mathrm{m}$, thereby preventing uniform focusing of the laser onto the surface. The nonlinear dependence of the ablation efficiency on laser intensity accentuates this problem. The sample surface roughness problem should be solvable with better sample preparation techniques. Another reason for the lack of high spatial resolution might be that the micro-focused laser beam concentrated the energy into a very small volume, causing most desorbed species to fragment, thereby reducing the molecular ion intensities.
The fs-LDPI-MS images shown in Figure 4 were collected using a $200 \mathrm{~mm}$ focal length achromatic doublet lens to focus the ultrafast laser onto the sample at a $30^{\circ}$ angle of incidence. The beam profile produced with this lens was found to display $\sim 25 \mu \mathrm{m}$ diameter focus when measured by the scanning knife edge method. ${ }^{21}$ The focusing optics worked well with the current biofilm and other biological samples as its Rayleigh range was on the millimeter scale, precluding the aforementioned focusing problems.

An artifact that can potentially cause false identification in LDPI mode arises from the two lasers firing in sequence with 5-100 $\mu$ s delay which may cause interference between ion packets, even if most of the direct ions are electrostatically rejected by the ion optical deflector. Both lasers generate an ion packet with an intervening delay comparable to the laser firing delay. The typical ion time-of-flight was $1-100 \mu \mathrm{s}$, so heavier direct ions produced by the fs desorption laser pulse could arrive at the detector together with lighter photoions produced by VUV ionization. This phenomenon can cause multiple broad peaks to appear in the low mass region of the LDPI mass spectra. These false peaks may be identified; however, by changing the delay between the two lasers to shift the LDI peaks with respect to those from LDPI. Alternatively, blocking the VUV beam eliminates photoions and leaves behind the undesirable LDI peaks. However, LDI does not typically generate many heavy ions, so these false peak artifacts do not frequently arise in LDPI-MS. Furthermore, all the LDPI data presented in this paper were checked to eliminate LDI artifacts.

\section{CONCLUSIONS}

Sub-100 fs laser pulses display a remarkable ability to machine materials with high precision while causing minimal damage to the remaining sample. ${ }^{1-14}$ Furthermore, ablation of intact biofilms and animal tissue with fs laser pulses previously demonstrated the feasibility of depth profiling via MS reanalysis at the same spot. ${ }^{7,15}$ The combination of VUV single photon ionization with fs laser desorption is shown here to permit MS imaging and depth profiling of intact biofilms deposited on various biomaterials without any addition of a matrix. In contrast, MALDI-MS imaging (as most commonly performed with UV ns lasers) does not permit depth profiling and requires the addition of a matrix. ${ }^{1,4,6}$ Nevertheless, a more thorough comparison with MALDI-MS and other methods awaits further studies as this work constitutes an early presentation of results for the recently constructed fs-LDPIMS instrument.

Mid-infrared ns lasers induce vibrationally resonant desorption, which in principle allows depth profiling and additionally avoids the need for matrix addition. ${ }^{1,39}$ However, the mid-IR laser desorption efficiency fluctuates with a sample's water content, ${ }^{40}$ so that the desorption efficiency is likely to vary dramatically at the interface between an aqueous biofilm or tissue and a non-aqueous biomaterial.

Femtosecond laser desorption without postionization has recently been shown to be effective for quantitative elemental analysis. ${ }^{21,41,42}$ Although elemental analysis was not pursued in the current work, both fs-LDI-MS and fs-LDPI-MS have been demonstrated with the current instrument. Quantitative analysis of small molecular species on a biomolecular interface was also previously demonstrated by ns-LDPI-MS imaging. ${ }^{34}$ It follows that spatially resolved, quantitative elemental and molecular analysis of biomaterial interfaces and intact biological 
samples should be possible with the MS imaging instrument described here by using appropriate fs laser fluences.

There are also some shortcomings to MS imaging using the current instrument. Although fs-LDI-MS imaging can rapidly collect images at a $1 \mathrm{kHz}$ rate, fs-LDPI-MS imaging is limited here by the $10 \mathrm{~Hz}$ repetition rate of the VUV-generating $\mathrm{Nd}$ :YAG laser. However, Nd:YAG lasers with a $200 \mathrm{~Hz}$ repetition rate are commercially available and could be deployed to increase the fs-LDPI-MS imaging rate by $20 \times$.

Another shortcoming of the current instrument is the lack of high mass resolution and tandem MS capabilities which precludes actually chemical identification of the MS peaks reported here. High resolution and tandem MS capabilities, despite their absence from most commercial SIMS instruments, ${ }^{2}$ are the hallmark of modern biological MS studies. Work is underway in the authors' laboratory to couple tandem MS to fs laser desorption to improve the capability of fs laser desorption-based MS for molecular identification.

SIMS remains the MS imaging method of choice for highest spatial resolution and depth profiling, although these capabilities come at the cost of molecular fragmentation that is sometimes quite severe. ${ }^{2,5}$ Insufficient spatial resolution is a problem for all methods in laser desorption-based methods for MS imaging. Generally, spatial resolution in MS imaging performed by ns UV or mid-IR lasers is limited to $\sim 50 \mu \mathrm{m}$, ${ }^{1,5}$ although $\sim 5 \mu \mathrm{m}$ spatial resolution has been reported for selected samples. ${ }^{43}$ Approximately 10 micrometer spatial resolution has been demonstrated in elemental analysis of inorganic test targets by fs-LDI-MS, ${ }^{21}$ and the highly nonlinear nature of the desorption event supports arguments that yet higher spatial resolution should be possible with fs-LDPI-MS and fs-LDI-MS. ${ }^{7}$ Two phenomena have limited the collection of higher spatial resolution MS images from biofilms by fs-LDI and fs-LDPI-MS. First is the problem of sample roughness; the highly nonlinear dependence of fs laser desorption on laser intensity requires that the sample roughness be well below the desired spatial resolution. Second is the problem of low postionization yields in fs-LDPI-MS, which limits the pixel size. Ongoing studies are examining strategies to overcome these two problems with the ultimate goal of achieving submicrometer spatial resolution with fs laser desorption.

\section{ASSOCIATED CONTENT}

\section{S Supporting Information}

Figure S1, in-house built photoelectron current detector; Figure S2, photoelectron current measured by $50 \mathrm{Ohms}$ terminated oscilloscpe; Figure S3, gas phase, $10.5 \mathrm{eV}$ single photon ionization mass spectrum from calibration mixture; Figure S4, demonstration of optical imaging on instrument; Figure S5, illustration of the gas frequency tripling cell optical layout and how the $355 \mathrm{~nm}$ beam was blocked. This material is available free of charge via the Internet at http://pubs.acs.org/.

\section{AUTHOR INFORMATION}

\section{Corresponding Author}

*E-mail: LHanley@uic.edu.

\section{Notes}

The authors declare no competing financial interest.

\section{ACKNOWLEDGMENTS}

The authors thank U.S. Customs Chicago for the donation of the Pegasus III mass spectrometer and colleagues in RJG's laboratory for maintenance of the ultrafast laser. They also thank the UIC Chemistry and Physics department machine shop and electronics shop for their construction of many customized parts as well as Jerry F. Moore for his useful input on the instrument design. The cultures for the chronic wound model biofilms were provided by Dr. G. James and colleagues at the Center for Biofilm Engineering at Montana State University.

\section{REFERENCES}

(1) Vertes, A.; Hitchins, V.; Phillips, K. S. Anal. Chem. 2012, 84, 3858-3866

(2) Fletcher, J. S.; Vickerman, J. C. Anal. Chem. 2013, 85, 610-639.

(3) Chughtai, K.; Heeren, R. M. A. Chem. Rev. 2010, 110, 32373277.

(4) Watrous, J. D.; Alexandrov, T.; Dorrestein, P. C. J. Mass Spectrom. 2011, 46, 209-222.

(5) Trouillon, R.; Passarelli, M. K.; Wang, J.; Kurczy, M. E.; Ewing, A. G. Anal. Chem. 2012, 85, 522-542.

(6) Blaze, M.T., M.; Aydin, B.; Carlson, R. P.; Hanley, L. Analyst

2012, 137, 5018-5025.

(7) Milasinovic, S.; Liu, Y.; Bhardwaj, C.; Blaze, M.T., M.; Gordon, R. J.; Hanley, , L. Anal. Chem. 2012, 84, 3945-3951.

(8) Dreisewerd, K. Chem. Rev. 2003, 103, 395-425.

(9) Knochenmuss, R.; Zhigilei, L. V. J. Mass Spectrom. 2010, 45, 333346.

(10)Trimpin, S.; Wang, B.; Inutan, E. D.; Li, J.; Lietz, C. B.; Harron, A.; Pagnotti, V. S.; Sardelis, D.; McEwen, C. N. J. Am. Soc. Mass Spectrom. 2012, 23, 1644-1660.

(11)Vogel, A.; Noack, J.; Huttman, G.; Paltauf, G. Appl. Phys. B: Lasers Opt. 2005, 81, 1015-1045.

(12)Toyran, S.; Liu, Y.; Singha, S.; Shan, S.; Cho, M. R.; Gordon, R. J.; Edwards, D. P. Exp. Eye Res. 2005, 81, 298-305.

(13)Liu, Y.; Sun, S.; Singha, S.; Cho, M. R.; Gordon, R. J. Biomaterials 2005, 26, 4597-4605.

(14)Nakamura, H.; Liu, Y.; Witt, T. E.; Edward, D. P.; Gordon, R. J. Invest. Ophthalmol. Visual Sci. 2009, 50, 1198-1204.

(15)Milasinovic, S.; Liu, Y.; Gasper, G. L.; Zhao, Y.; Johnston, J. L.; Gordon, R. J.; Hanley, L. J. Vac. Sci. Technol., A 2010, 28, 647-651.

(16)Berry, J. I.; Sun, S.; Dou, Y.; Wucher, A.; Winograd, N. Anal.

Chem. 2003, 75, 5146-5151.

(17)Wichmann, J. M.; Lupulescu, C.; Wöste, L.; Lindinger, A. Rapid Comm. Mass Spectrom. 2009, 23, 1105-1108.

(18)Coello, Y.; Gunaratne, T. C.; Dantus, M. Proc. SPIE 2009, 7182, $71821 \mathrm{~W}-71825$.

(19)Kalcic, C. L.; Gunaratne, T. C.; Jones, A. D.; Dantus, M.; Reid, G. E. J. Am. Chem. Soc. 2009, 131, 940-942.

(20)Coello, Y.; Jones, A. D.; Gunaratne, T. C.; Dantus, M. Anal. Chem. 2010, 82, 2753.

(21)Cui, Y.; Moore, J. F.; Milasinovic, S.; Liu, Y.; Gordon, R. J.; Hanley, L. Rev. Sci. Instrum. 2012, 83, 093702.

(22)Brady, J. J.; Judge, E. J.; Levis, R. J. Rapid Comm. Mass Spectrom. 2009, 23, 3151-3157.

(23)Judge, E. J.; Brady, J. J.; Dalton, D.; Levis, R. J. Anal. Chem. 2010, 82, 3231 .

(24)Judge, E. J.; Brady, J. J.; Barbano, P. E.; Levis, R. J. Anal. Chem.

2011, 83, 2145-2151.

(25)Perez, J. J.; Flanigan, P. M.; Brady, J. J.; Levis, R. J. Anal. Chem. 2012, 85, 296-302.

(26)Hanley, L.; Kornienko, O.; Ada, E. T.; Fuoco, E.; Trevor, J. L. J. Mass Spectrom. 1999, 34, 705-723.

(27)Edirisinghe, P. D.; Moore, J. F.; Calaway, W. F.; Veryovkin, I. V.; Pellin, M. J.; Hanley, L. Anal. Chem. 2006, 78, 5876-5883.

(28)Edirisinghe, P. D.; Moore, J. F.; Skinner-Nemec, K. A.; Lindberg, C.; Giometti, C. S.; Veryovkin, I. V.; Hunt, J. E.; Pellin, M. J.; Hanley, L. Anal. Chem. 2007, 79, 508-514.

(29)Hanley, L.; Zimmermann, R. Anal. Chem. 2009, 81, 4174-4182. 
(30) Akhmetov, A.; Moore, J. F.; Gasper, G. L.; Koin, P. J.; Hanley, L. J. Mass Spectrom. 2010, 45, 137-145.

(31) Gasper, G. L.; Takahashi, L. K.; Zhou, J.; Ahmed, M.; Moore, J. F.; Hanley, L. Anal. Chem. 2010, 82, 7472-7478.

(32) Gasper, G. L.; Takahashi, L. K.; Zhou, J.; Ahmed, M.; Moore, J. F.; Hanley, L. Nucl. Instrum. Methods Phys. Res., Sect. A 2011, 649, 222-224.

(33) Blaze, M.T., M.; Takahashi, L. K.; Zhou, J.; Ahmed, M.; Gasper, G. L.; Pleticha, F. D.; Hanley, L. Anal. Chem. 2011, 83, 4962-4969.

(34) Blaze, M.T., M.; Akhmetov, A.; Aydin, B.; Edirisinghe, P. D.; Uygur, G.; Hanley, L. Anal. Chem. 2012, 84, 9410-9415.

(35) Gasper, G. L.; Carlson, R.; Akhmetov, A.; Moore, J. F.; Hanley, L. Proteomics 2008, 8, 3816-3821.

(36) Bhardwaj, C.; Moore, J. F.; Cui, Y.; Gasper, G. L.; Bernstein, H. C.; Carlson, R. P.; Hanley, L. Anal. Bioanal. Chem. 2012, http://dx.doi. org/10.1007/s00216-012-6454-0.

(37) Dyer, P. E.; Monk, P.; Snelling, H. V.; Walton, C. D. Appl. Surf. Sci. 2002, 186, 206-210.

(38) Woods, J.; Boegli, L.; Kirker, K. R.; Agostinho, A. M.; Durch, A. M.; deLancey Pulcini, E.; Stewart, P. S.; James, G. A. J. Appl. Microbiol. 2012, 112, 998-1006.

(39) Galhena, A. S.; Harris, G. A.; Nyadong, L.; Murray, K. K.; Fernandez, F. M. Anal. Chem. 2010, 82, 2178-2181.

(40) Chen, Z.; Vertes, A. Phys. Rev. E 2008, 77, 036316.

(41) Zhang, B.; He, M.; Hang, W.; Huang, B. Anal. Chem. 2013, 85, 4507-4511.

(42) Gao, Y.; Lin, Y.; Zhang, B.; Zou, D.; He, M.; Dong, B.; Hang, W.; Huang, B. Anal. Chem. 2013, 85, 4268-4272.

(43) Zavalin, A.; Todd, E. M.; Rawhouser, P. D.; Yang, J.; Norris, J. L.; Caprioli, R. M. J. Mass Spectrom. 2012, 47, 1473-1481. 